\title{
Acquired Hemophilia A Associated with Dipeptidyl Peptidase-4 Inhibitors for the Treatment of Type 2 Diabetes Mellitus: A Single-Center Case Series in Japan
}

\author{
Satoshi Yamasaki - Masanori Kadowaki - Takashi Jiromaru • \\ Ken Takase $\cdot$ Hiromi Iwasaki
}

Received: February 21, 2019 / Published online: March 29, 2019

(C) The Author(s) 2019

\begin{abstract}
Herein we describe four patients with acquired hemophilia A (AHA) caused by factor VIII (FVIII) inhibitor and histories of dipeptidyl peptidase-4 inhibitor (DPP4-I) treatment for diabetes mellitus (DM). Drug exposure can cause a breakdown of immune tolerance to FVIII associated with CD4 $\mathrm{T}$ cells, resulting in the induction of autoantibodies against FVIII. In patient 1 in the present series, FVIII inhibitor disappeared after DPP4-I treatment. The DPP4-I treatment was stopped faster in patient 1 than it was in patient 2, whose FVIII inhibitor titer was higher than patient 1 's. Two patients died: patient 3 due to brain infarction after recurrence associated with the development of sigmoid colon rupture, and patient 4 due to multiple organ failure associated with
\end{abstract}

Enhanced Digital Features To view enhanced digital features for this article go to https://doi.org/10.6084/ m9.figshare.7873700.

Electronic supplementary material The online version of this article (https://doi.org/10.1007/s13300019-0609-3) contains supplementary material, which is available to authorized users.

S. Yamasaki ( $)$ · M. Kadowaki · T. Jiromaru .

K. Takase $\cdot$ H. Iwasaki

Department of Hematology and Clinical Research Institute, National Hospital Organization Kyushu

Medical Center, 1-8-1 Jigyohama, Chuo-Ku,

Fukuoka 810-8563, Japan

e-mail: yamas009@gmail.com
Clostridium difficile colitis. DPP4-I treatment may create an ideal environment for the induction of new antibodies and AHA onset associated with tumor necrosis factor- $\alpha$ reduction. These are the first reported cases of the potential development and/or prolonging of AHA after DDP4-I treatment for DM, and they suggest possible disease associations.

Keywords: Acquired hemophilia A; Diabetes mellitus; Dipeptidyl peptidase-4 inhibitors; Tumor necrosis factor- $\alpha$

\section{INTRODUCTION}

Acquired hemophilia A (AHA) is caused by autoantibodies that inhibit factor VIII (FVIII) activity and lead to life-threatening hemorrhage [1]. It can be associated with autoimmune conditions, underlying malignancies, or drug allergies, but no specific cause is identified in an estimated $50 \%$ of patients $[2,3]$. Diabetes mellitus (DM) is a highly prevalent condition diagnosed via well-established criteria, but the main causes of DM have not been well characterized. Genetics, environmental factors, poor diet, obesity, medications, infections, and a sedentary lifestyle are variably associated with DM in some cases [4]. Dipeptidyl peptidase-4 inhibitors (DPP4-Is) are relatively new oral antidiabetic drugs that have various effects on 
the immune system, including plasma tumor necrosis factor (TNF)- $\alpha$ reduction [5] and the inhibition of pathogenic T cells [6]. In patients with type 2 DM, DDP4-Is reportedly reduced the risk of rheumatoid arthritis [7] but increased the risk of inflammatory bowel disease [8]. Currently, there are no reports on associations between AHA and DDP4-I administration in type $2 \mathrm{DM}$ patients.

\section{CASES}

Herein we describe four patients with AHA and histories of DDP4-I treatment for DM (Table 1). Two were male and two were female, and their ages ranged from 61 to 84 years. Activated partial thromboplastin times (APTTs) ranged from 71.1 to $115.5 \mathrm{~s}$ (normal range $=21-33 \mathrm{~s}$ ), which is markedly prolonged (see figures in the Electronic supplementary material, ESM). Anemia progressed to $5.2-6.0 \mathrm{~g} / \mathrm{dL}$ of hemoglobin (normal range $=13.5-17.0 \mathrm{~g} / \mathrm{dL}$ ), necessitating red blood cell transfusions of 16-24 units. FVIII activity levels were $<1 \%$ in all cases (normal range $=60-150 \%$, and FVIII inhibitor titers ranged from 14 to 100 Bethesda units $/ \mathrm{mL}$ (normal titer $=0$ Bethesda units $/ \mathrm{mL}$ ). Serum glycoprotein levels ranged from 15 to $20 \%$ (normal range $=12.4-16.3 \%$ ) and all patients received a DDP4-I (sitagliptin phosphate hydrate in three cases and alogliptin benzonate in one case). Due to limitations on the use of FVIII imposed by the Japanese health insurance system, AHA patients can receive recombinant activated factor VII but not FVIII. Therefore, recombinant activated factor VII was administered to all four patients (three at diagnosis, one at recurrence) to control bleeding after bleeding diathesis, an isolated prolonged APTT, detection of low coagulation factor activity in mixing studies, or failure to exhibit APTT normalization after mixing of the patient's serum with a nonhemophiliac donor's serum. Mixing studies are performed to determine the presence of factor deficiency or factor inhibitors, or the presence of nonspecific inhibitors such as lupus anticoagulants, if APTT is prolonged in the specimen. With regard to immunological treatments for the eradication of inhibitors, prednisolone was administered to all four patients, cyclophosphamide was administered to patient 1 , and rituximab was administered to patient 4 . In patient 1 , FVIII inhibitor disappeared after DPP4-I treatment. The DPP4-I treatment was stopped faster in patient 1 than it was in patient 2, whose FVIII inhibitor titer was higher than patient 1 's. In patient 2, FVIII inhibitor decreased from 27 Bethesda units $/ \mathrm{mL}$ to 1 Bethesda unit/mL with the use of recombinant activated factor VII and the administration of prednisolone (daily dose of $1 \mathrm{mg}$ / $\mathrm{kg}$ /day), and hemostasis was achieved. Prednisolone was tapered off and the FVIII inhibitor titer was negative after approximately 18 months. Two patients died: patient 3 due to brain infarction after recurrence associated with the development of sigmoid colon rupture, and patient 4 due to multiple organ failure associated with Clostridium difficile colitis. The present case series was retrospective and did not involve any experimental intervention. Accordingly, no institutional ethical approval was required or sought. Informed consent was obtained from all individual participants included in the study.

\section{DISCUSSION}

AHA is characterized by the presence of an autoimmune mechanism that either alone or accompanied by autoimmune disease, aging, or drug exposure causes a breakdown in immune tolerance to FVIII autoantibodies involving CD4 $\mathrm{T}$ cells and results in the development of autoantibodies against FVIII. DPP4-I may be responsible for the impaired proliferative response of CD4 T cells to antigens and mitogens observed in hemophiliacs [9]. Although immunosuppressive treatment should eradicate inhibitors as soon as a diagnosis of AHA is confirmed, immunosuppression is associated with significant side effects including sepsis and colitis in older DM patients. TNF- $\alpha$ inhibitors may activate autoimmune reactions and the production of autoantibodies, and in $2 / 4$ of the present cases AHA may have been induced by TNF- $\alpha$ inhibitors, as has previously been reported [10, 11]. DPP4-I treatment may create an ideal environment for new antibody formation 


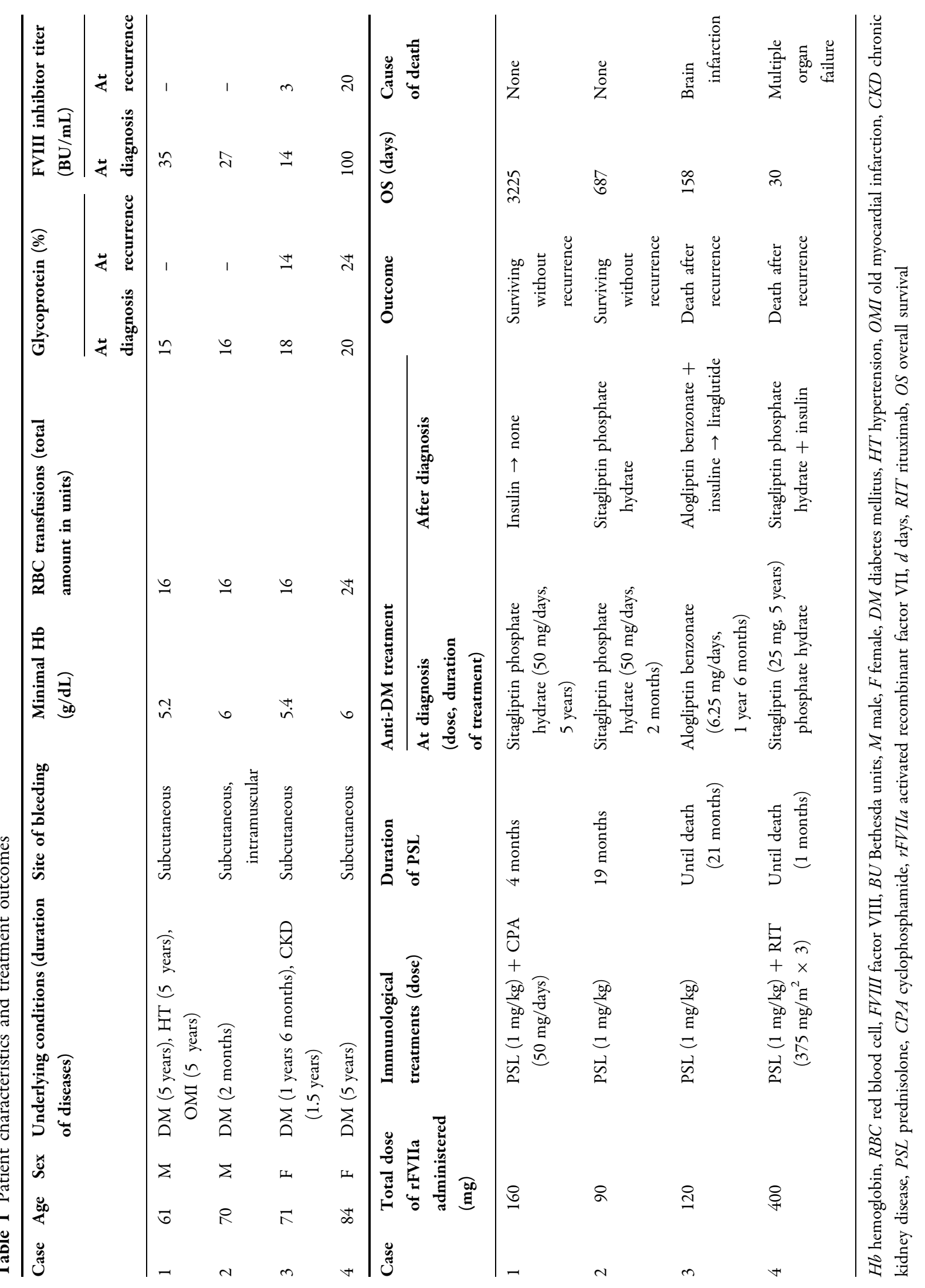


and AHA onset associated with TNF- $\alpha$ reduction.

Cases of the onset of AHA associated with hypersensitivity to drugs such as antibiotics (penicillin, sulfonamides, and chloramphenicol), anticonvulsants (phenytoin), antihypertensive agents (methyldopa), and vaccination have been reported [12]. As increasing titers of FVIII inhibitors due to drug hypersensitivity disappear after termination of the responsible drug [13], immunological treatments to eradicate FVIII autoantibodies may not be required in patients who experience drug hypersensitivity.

While the observations in the present case series are suggestive of associations, there are substantial limitations to the conclusions that can be drawn from it. The study was a retrospective case series that only included four patients, and low patient numbers can hinder the recognition of small differences in outcomes. Notwithstanding this, the results are concordant with an association between AHA and DPP4-I. Current AHA therapeutic strategies in DM patients who have undergone DDP4-I administration may not improve outcomes in patients who are resistant to immunological treatments to eradicate FVIII autoantibodies. Because the analysis of various comorbidities in DM patients was beyond the scope of the current study, and the best immunological treatments to use in DM patients with AHA remain uncertain, carefully designed prospective trials are essential to determine the contribution of immunological treatments to the successful eradication of FVIII autoantibodies. Another consideration that limits the interpretation of the present study is a lack of knowledge pertaining to predisposing factors for AHA. Lastly, a longer follow-up period may be needed to better evaluate eligibility criteria for DDP4-I administration in DM patients with AHA.

In conclusion, data derived from the present case series suggest that DDP4-I treatment for DM may induce and/or prolong AHA. Prospective observations and studies with larger numbers of patients are needed to improve the safety and efficacy of DDP4-I treatment in DM patients with AHA.

\section{ACKNOWLEDGEMENTS}

We thank the patients and clinical staff for their participation in the study.

Funding. No funding or sponsorship was received for this study or publication of this article. The article processing charges were funded by the authors.

Editorial Assistance. The authors acknowledge The Clinical Research Institute, Kyushu Medical Hospital for editorial support. The authors also thank Dr. Owen Proudfoot from Edanz Group (http://www.edanzediting.com/ ac) for editing a draft of this manuscript. Editorial assistance was funded by the authors.

Authorship. All named authors meet the International Committee of Medical Journal Editors criteria for authorship of this article, take responsibility for the integrity of the work as a whole, and have given their approval for this version to be published.

Authorship Contributions. Satoshi Yamasaki designed the study, analyzed the data, and prepared the manuscript. Masanori Kadowaki, Takashi Jiromaru, Ken Takase, and Hiromi Iwasaki prepared and reviewed the manuscript. All authors have approved the final manuscript.

Disclosures. Satoshi Yamasaki, Masanori Kadowaki, Takashi Jiromaru, Ken Takase and Hiromi Iwasaki have nothing to disclose.

Compliance with Ethics Guidelines. The present case series was retrospective and did not involve any experimental intervention. Accordingly, no institutional ethical approval was required or sought. Informed consent was obtained from all individual participants included in the study.

Open Access. This article is distributed under the terms of the Creative Commons Attribution-NonCommercial 4.0 International License (http://creativecommons.org/licenses/ by-nc/4.0/), which permits any noncommercial use, distribution, and reproduction in any 
medium, provided you give appropriate credit to the original author(s) and the source, provide a link to the Creative Commons license, and indicate if changes were made.

\section{REFERENCES}

1. Kruse-Jarres R, Kempton CL, Baudo F, Collins PW, Knoebl P, Leissinger CA, Tiede A, Kessler CM. Acquired hemophilia A: updated review of evidence and treatment guidance. Am J Hematol. 2017;92:695-705.

2. Baudo F, Collins $P$, Huth-Kühne A, Lévesque $H$, Marco P, Nemes L, Pellegrini F, Tengborn L, Knoebl $\mathrm{P}, \mathrm{EACH} 2$ Registry Contributors. Management of bleeding in acquired hemophilia A: results from the European Acquired Haemophilia (EACH2) Registry. Blood. 2012;120:39-46.

3. Shetty S, Bhave M, Ghosh K. Acquired hemophilia A: diagnosis, aetiology, clinical spectrum and treatment options. Autoimmun Rev. 2011;10:311-6.

4. American Diabetes Association. 2. Classification and diagnosis of diabetes: standards of medical care in diabetes-2018. Diabetes Care. 2018;41(Suppl 1):S13-27.

5. Atkin SL, Katsiki N, Banach M, Mikhailidis DP, Pirro M, Sahebkar A. Effect of dipeptidyl peptidase-4 inhibitors on circulating tumor necrosis factor- $\alpha$ concentrations: a systematic review and metaanalysis of controlled trials. J Diabetes Complicat. 2017;31:1458-64.

6. Zhao Y, Yang L, Wang X, Zhou Z. The new insights of DPP-4 inhibitors: their potential immune modulatory function in autoimmune diabetes. Diabetes Metab Res Rev. 2014;30:646-53.

7. Kim SC, Schneeweiss S, Glynn RJ, Doherty M, Goldfine AB, Solomon DH. Dipeptidyl peptidase- 4 inhibitors in type 2 diabetes may reduce the risk of autoimmune diseases: a population-based cohort study. Ann Rheum Dis. 2015;74:1968-75.

8. Abrahami D, Douros A, Yin H, Yu OHY, Renoux C, Bitton A, Azoulay L. Dipeptidyl peptidase-4 inhibitors and incidence of inflammatory bowel disease among patients with type 2 diabetes: population based cohort study. BMJ. 2018;360:k872.

9. Invernizzi R, Montani N, Giusto $\mathrm{M}$, Mosconi $\mathrm{E}$, Lorenzutti F, Comolli G, Pecci A, Gamba G. Expression of dipeptidylaminopeptidase IV/CD26 in peripheral blood lymphocytes of hemophilic subjects. Eur J Haematol. 1998;60:145-52.

10. Arthanari S, Ahmad H, Nisar M. Fatal acquired hemophilia A in a patient with rheumatoid arthritis treated with adalimumab. J Clin Rheumatol. 2012;18:50-1.

11. Banse $\mathrm{C}$, Benhamou $\mathrm{Y}$, Lequerré $\mathrm{T}$, Le Cam-Duchez $\mathrm{V}$, Lévesque $\mathrm{H}$, Vittecoq $\mathrm{O}$. Acquired hemophilia possibly induced by etanercept in a patient with rheumatoid arthritis. Jt Bone Spine. 2015;82:200-2.

12. Franchini M, Gandini G, Di Paolantonio T, Mariani G. Acquired hemophilia A: a concise review. Am J Hematol. 2005;80:55-63.

13. Delgado J, Jimenez-Yuste V, Hernandez-Navarro F, Villar A. Acquired haemophilia: review and metaanalysis focused on therapy and prognostic factors. Br J Haematol. 2003;121:21-35. 